\title{
ON THE EXPECTED VALUE OF UNCERTAIN VARIABLES
}

Received : 7/12/2017

Accepted : 18/1/2018

\section{Zainab Hayder Abid AL-Aali}

Department of Mathematics and Computer applications, College of Science, Muthanna University

E-mail address: zhayder49@gmail.com

\section{$\underline{\text { Abstract }}$}

In this work, some mathematical properties and several inequalities for uncertain variables that based on uncertain measure and expected value are proved. Finally the relation between convergence in $p$ th moment and convergence in measure for uncertain sequence were investigated.

Keyword: Uncertain measure, uncertain variable, expected value.

\section{1-Introduction}

Liu [2] in 2007 founded uncertainty theory, and Liu [8] refined it in 2010 . The first basic concepts of uncertainty theory is an uncertain measure which defined as a set function from $M: F \rightarrow R$, satisfies the following axioms:

(A1) (Normality) : $M(\Omega)=1$.

(A2)(Monotonicity ) $: M(A) \leq M(B)$ whenever $A \subset B$.

(A3)(Self-duality ): $M(A)+M\left(A^{c}\right)=1$ for any event $A$.

(A4)(Countable Subadditivity): For every countable sequence of events $\left\{A_{i}\right\}$, we have $M\left(\mathrm{Y}_{i=1}^{\infty} A_{i}\right) \leq \sum_{i=1}^{\infty} M\left(A_{i}\right)$

(A5) (Product Measure Axiom): Let $\left(\Omega_{k}, F_{k}, M_{k}\right)$ be uncertainty spaces for $k=1,2, \Lambda, n$. Then the product uncertain measure $M$ is an uncertain measure on the product $\sigma$-filed $F_{1} \times F_{2} \times \Lambda \times F_{n}$ satisfying:

\section{Definition (1-1) [3]}

The triple $(\Omega, F, M)$ is called an uncertainty space which $\Omega$ be a nonempty set, $F$ be a $\sigma$ field over $\Omega$, and $M$ be an uncertain measure.

\section{Definition (1-2) [1]}

An uncertain variable $X$ defined as measurable function from an uncertainty space
$M\left(\prod_{k=1}^{n} A_{k}\right)=\min \left\{M_{k}\left(A_{k}\right): A_{k} \in F_{k}, k=1,2, \ldots . n\right\}$

That is, for each event $A \in F=F_{1} \times F_{2} \times \Lambda \times F_{n}$, we have

$M(A)=\left\{\begin{array}{cc}\alpha, & \alpha>0.5 \\ 1-\beta, & \beta>0.5 \\ 0.5, & \text { o.w }\end{array}\right.$

where

$\alpha=\sup \left\{\begin{array}{l}\left.\min \left\{M_{k}\left(A_{k}\right)\right\}: k=1,2, \Lambda, n\right\}: \\ A_{1} \times A_{2} \times \Lambda \times A_{n} \subseteq A\end{array}\right\}$
$\beta=\sup \left\{\begin{array}{l}\left.\min \left\{M_{k}\left(A_{k}\right)\right\}: k=1,2, \Lambda, n\right\}: \\ A_{1} \times A_{2} \times \Lambda \times A_{n} \subseteq A^{c}\end{array}\right\}$

to the set of real numbers such that $\{X \in B\}$ is an event for any set $B$ of $\mathfrak{R}$ 


\section{Definition (1-3) [7]}

Assume that $X_{1}, X_{2}, \ldots, X_{n}$ be an uncertain variables. We say that $X_{i}, i=1,2, \ldots, n$ be an independent uncertain variables if Definition(1-4)[10]

Assume that $\left\{x_{n}\right\}$ be an uncertain variables sequence .we say that $\left\{x_{n}\right\}$ convergence in $p$ th moment to uncertain variable $x$ if $\lim _{n \rightarrow \infty} E\left\{\left|x_{n}-x\right|^{p}\right\}=0$ for any $p \in[1, \infty)$. we

\section{Definition(1-5)[9]}

Assume that $\left\{x_{n}\right\}$ be an uncertain variables sequence. we say that $\left\{x_{n}\right\}$ is convergence in

\section{Theorem (1-6) [9]}

Assume that $X$ be an uncertain variable.

Then we get $M(|X| \geq \varepsilon) \leq \frac{E\left(|X|^{p}\right)}{\varepsilon^{p}}$, for any given numbers $\varepsilon>0$ and $p>0$.

\section{Theorem (1-7)[5]}

Assume that $X$ and $Y$ be two independent uncertain variables such that $E\left(|X|^{p}\right)$ and $E\left(|Y|^{p}\right) \quad$ are finite. Then we get $E(X Y) \leq \sqrt[p]{E\left(|X|^{p}\right)}+\sqrt[q]{E\left(|Y|^{q}\right)}, \quad$ for $\quad$ any

\section{Theorem (1-8)[5]}

Assume that $X$ and $Y$ be two independent uncertain variables such that $E\left(|X|^{p}\right)$ and $E\left(|Y|^{p}\right) \quad$ are finite. Then we get

\section{$\underline{\text { 2- Expected value of uncertain variables }}$}

\section{Definition (2-1) [6,8]}

The expected value operator of uncertain variable $X$ is defined by

$E(X)=\int_{0}^{+\infty} M(X \geq r) d r-\int_{-\infty}^{0} M(X \leq r) d r$,

provided that at least one of the two integrals is
$M\left(\mathrm{I}_{i=1}^{n}\left(X_{n} \in B_{i}\right)\right)=\min _{1 \leq i \leq n} M_{i}\left(X_{i} \in B_{i}\right), \quad$ for any Borel $B_{1}, B_{2}, \ldots, B_{n}$ of $\mathfrak{R}$.

say that $\left\{x_{n}\right\}$ convergence in mean to uncertain variable $x$ whenever $p=1$ and we say that $\left\{x_{n}\right\}$ convergence in mean square to uncertain variable $x$ whenever $p=2$.

measure to uncertain variable $x$ if $\lim _{n \rightarrow \infty} M\left\{\left|x_{n}-x\right| \geq \varepsilon\right\}=0$, for every $\varepsilon>0$.

positive numbers $p$ and $q$ such that $\frac{1}{p}+\frac{1}{q}=1$.

$\sqrt[p]{E\left(|X+Y|^{p}\right)} \leq \sqrt[p]{E\left(|X|^{p}\right)}+\sqrt[p]{E\left(|Y|^{p}\right)}$ for any real number $p$ such that $p \geq 1$.

finite. The variance of $X$ is defined by $E(X)=E(X-e)^{2} \quad$ where $X$ is the finite expected value of $X$. Generally, the expected value $E\left((X)^{k}\right)$ is called the $k$ th absolute 
moment of the uncertain variable $X$ for any positive integer $k$.

\section{Theorem(2-2) [5]}

Assume that $X$ and $Y$ be two independent uncertain variables such that $E(X)<\infty$, and $E(Y)<\infty$. Then we get

\section{Theorem(2-3)}

Assume that $X$ be an uncertain variable. Then

$$
E(-X)=-E(X) \text {. }
$$

\section{Proof:}

By using definition (2-1), then we get

$$
\begin{aligned}
& E(-X)=\int_{0}^{+\infty} M(-X \geq r) d r-\int_{-\infty}^{0} M(-X \leq r) d r \\
& =\int_{0}^{+\infty} M(X \leq-r) d r-\int_{-\infty}^{0} M(X \geq-r) d r
\end{aligned}
$$

\section{Theorem(2-4)}

Assume that $X$ and $Y$ be two an independent uncertain variables such that $E(|X|)<\infty$, and

\section{Proof:}

By using theorem (2-2), then we get $E(|X+Y|) \leq E(|X|+|Y|)=E(|X|)+E(|Y|)$

\section{3- Main Results}

\section{Theorem(3-1)}

Assume that $X$ and $Y$ be two independent uncertain variables, and $f: R^{2} \rightarrow R$ a convex function such that $E(X)<\infty$, and $E(Y)<\infty$. Then

$$
f(E(X), E(Y)) \leq E(f(X, Y))
$$

\section{Proof:}

Since $f: R^{2} \rightarrow R \quad$ is a convex function, then for any given $\left(x_{1}, y_{1}\right) \in R^{2}$, then there exist $a_{1}, a_{2} \in R$ such that

$f(x, y)-f\left(x_{1}, y_{1}\right) \geq a_{1}\left(x-x_{1}\right)+a_{2}\left(y-y_{1}\right)$, for all $x, y \in R$

Put $x_{1}=E(X), y_{1}=E(Y)$ and $x=X, y=Y$

Then, $f(X, Y)-f(E(X), E(Y))$

$$
\begin{aligned}
& E(|Y|)<\infty . \quad \text { Then } \\
& E(|X+Y|) \leq E(|X|)+E(|Y|)
\end{aligned}
$$$$
\text { we }
$$

\section{et}

$$
\begin{aligned}
& =\int_{-\infty}^{0} M(X \leq r) d r-\int_{0}^{+\infty} M(X \geq r) d r \\
& =-\left[\int_{0}^{+\infty} M(X \geq r) d r-\int_{-\infty}^{0} M(X \leq r) d r\right] \\
& =-E(X)
\end{aligned}
$$

$$
\begin{aligned}
& E[f(X, Y)-f(E(X), E(Y))] \\
& \geq E\left[a_{1}(X-E(X))+a_{2}(Y-E(Y))\right]
\end{aligned}
$$

From theorem (2-2), we get $E(f(X, Y))-f(E(X), E(Y)) \geq$$$
a_{1}(E(X)-E(X))+a_{2}(E(Y)-E(Y))=0
$$$$
f(E(X), E(Y)) \leq E(f(X, Y))
$$ 


\section{Theorem(3-2)}

Assume that $X$ and $Y$ be two an independent uncertain variables such that $E\left(|X|^{p}\right)<\infty$ and $E\left(|Y|^{p}\right)<\infty$, where $p>0$.

Then $E\left(|X+Y|^{p}\right) \leq C E\left(|X|^{p}\right)+C E\left(|Y|^{p}\right)$.

\section{Proof:}

for any $a_{1}, a_{2} \in \mathfrak{R}$, we get

$\left|a_{1}+a_{2}\right|^{p} \leq C\left|a_{1}\right|^{p}+C\left|a_{2}\right|^{p}$

Put $a_{1}=X$ and $a_{2}=Y$, we get

$|X+Y|^{p} \leq C|X|^{p}+C|Y|^{p}$

\section{Theorem (3-3)}

Assume that $\left\{x_{n}\right\}$ be an uncertain variables sequence and $x, y$ be two uncertain variables . If $x_{n} \rightarrow x$ as $n \rightarrow \infty$ (in $p$ th moment), and

\section{Proof:}

$$
\begin{aligned}
& \text { Since } \quad \lim _{n \rightarrow \infty} E\left\{\left|x_{n}-x\right|^{p}\right\}=0 \quad \text { and } \\
& \lim _{n \rightarrow \infty} E\left\{\left|x_{n}-y\right|^{p}\right\}=0
\end{aligned}
$$

By using Theorem (3-2) that

\section{Theorem (3-4)}

Assume that $\left\{x_{n}\right\},\left\{y_{n}\right\}$ be two uncertain variables sequence and $x, y$ be two uncertain variables. If $x_{n} \rightarrow x$ as $n \rightarrow \infty \quad$ (in $p$ th moment), and $y_{n} \rightarrow y \quad$ as $n \rightarrow \infty \quad$ (in $p$ th Proof:

Since $\quad \lim _{n \rightarrow \infty} E\left\{\left|x_{n}-x\right|^{p}\right\}=0 \quad$ and $\lim _{n \rightarrow \infty} E\left\{\left|y_{n}-y\right|^{p}\right\}=0$

By using Theorem (3-2) that
Holds, where $C=\left\{\begin{array}{cc}1 & , \text { if } 0<p \leq 1 \\ 2^{p-1} & , \text { ifp }>1\end{array}\right\}$ $E\left[|X+Y|^{p}\right] \leq E\left[C|X|^{p}+C|Y|^{p}\right]$, by theorem (2-2), then get $E\left(|X+Y|^{p}\right) \leq C E\left(|X|^{p}\right)+C E\left(|Y|^{p}\right)$ $x_{n} \rightarrow y$ as $n \rightarrow \infty$ (in $p$ th moment), Then $x=y$.

$$
\begin{aligned}
& E\left\{|x-y|^{p}\right\}=E\left\{\left|x-x_{n}+x_{n}-y\right|^{p}\right\} \\
& \leq C E\left\{\left|x_{n}-x\right|^{p}\right\}+C E\left[\left[y_{n}-\left.y\right|^{p}\right] \rightarrow 0\right.
\end{aligned},
$$$$
\text { as } \quad n \rightarrow \infty \text {. we get } E\left\{x-\left.y\right|^{p}\right\}=0 \text {. }
$$

moment), if $E\left\{\left|x_{n}-x\right|^{p}\right\}$ and $E\left\{y_{n}-\left.y\right|^{p}\right\}$ are independent for all $n$. Then $x_{n}-y_{n} \rightarrow x-y$ as $n \rightarrow \infty$.

$E\left\{\left(x_{n}-y_{n}\right)-\left.(x-y)\right|^{p}\right\}$

$=E\left\{\left(x_{n}-x\right)+\left.\left(y-y_{n}\right)\right|^{p}\right\}$ $\leq C E\left\{x_{n}-\left.x\right|^{p}\right\}+C E\left\{\left|y_{n}-y\right|^{p}\right\} \rightarrow 0$,

As $\quad n \rightarrow \infty, \quad$ then $\quad$ we get
$\lim _{n \rightarrow \infty} E\left\{\left(x_{n}-y_{n}\right)-\left.(x-y)\right|^{p}\right\}=0$. 


\section{Theorem (3-5)}

Assume that $\left\{x_{n}\right\}$ be an uncertain variables $x_{n} \rightarrow x \quad$ (in $p$ th moment), then sequence and $x$ be an uncertain variable. If $\lim _{n \rightarrow \infty} E\left\{\left|x_{n}\right|^{p}\right\}=E\left\{|x|^{p}\right\}$.

Proof:

There is two cases:

Case(1): If $0<p \leq 1$. Put $x_{n}=x+\left(x_{n}-x\right)$ By using theorem (1-8), that and $x=x_{n}+\left(x-x_{n}\right)$

$\left(E\left\{\left|x_{n}\right|^{p}\right\}\right)^{\frac{1}{p}} \leq\left(E\left\{\left.x\right|^{p}\right\}\right)^{\frac{1}{p}}+\left(E\left\{\left|x_{n}-x\right|^{p}\right\}\right)^{\frac{1}{p}}$

By using theorem (3-2), we get $E\left\{\left|x_{n}\right|^{p}\right\} \leq E\left\{|x|^{p}\right\}+E\left\{x_{n}-\left.x\right|^{p}\right\} \quad, \quad$ and $E\left\{|x|^{p}\right\} \leq E\left\{\left|x_{n}\right|^{p}\right\}+E\left\{x_{n}-\left.x\right|^{p}\right\}$, furthermore $\lim _{n \rightarrow \infty}\left(E\left\{\left|x_{n}\right|^{p}\right\}-E\left\{|x|^{p}\right\}\right) \leq \lim _{n \rightarrow \infty}\left(E\left\{\left|x_{n}-x\right|^{p}\right\}\right)=0$,

Which implies $\lim _{n \rightarrow \infty}\left(E\left\{\left|x_{n}\right|^{p}\right\}\right)=\left(E\left\{|x|^{p}\right\}\right)$

$\left(E\left\{|x|^{p}\right\}\right)^{\frac{1}{p}} \leq\left(E\left\{\left|x_{n}\right|^{p}\right\}\right)^{\frac{1}{p}}+\left(E\left\{\left|x_{n}-x\right|^{p}\right\}\right)^{\frac{1}{p}}$

Then we get

Case(2) : If $p>1$. Put $x_{n}=x+\left(x_{n}-x\right)$ and $x=x_{n}+\left(x-x_{n}\right)$

$\lim _{n \rightarrow \infty}\left(\left(E\left\{\left|x_{n}\right|^{p}\right\}\right)^{\frac{1}{p}}-\left(E\left\{\left.x\right|^{p}\right\}\right)^{\frac{1}{p}}\right) \leq \lim _{n \rightarrow \infty}\left(E\left\{x_{n}-\left.x\right|^{p}\right\}\right)^{\frac{1}{p}}=0$

Which implies $\lim _{n \rightarrow \infty}\left(E\left\{\left.x_{n}\right|^{p}\right\}\right)^{\frac{1}{p}}=\left(E\left\{\left.x\right|^{p}\right\}\right)^{\frac{1}{p}}$.

Since $f(x)=x^{\frac{1}{p}}$ is continuous function, thus $\lim _{n \rightarrow \infty} E\left\{\left|x_{n}\right|^{p}\right\}=E\left\{|x|^{p}\right\}$

\section{Theorem (3-6)}

Assume that $\left\{x_{n}\right\}$ and $\left\{y_{n}\right\}$ be two uncertain variables sequence and $x, y$ be two uncertain variables. If $x_{n} \rightarrow x$ as $n \rightarrow \infty$ (in $p$ th moment ), and $y_{n} \rightarrow y$ as $n \rightarrow \infty$ (in $q$ th moment), for any positive numbers $p$ and $q$ such that $\frac{1}{p}+\frac{1}{q}=1$, and $p>1$. Then $x_{n} y_{n} \rightarrow x y$ as $n \rightarrow \infty$ (in mean).

Proof:

$$
\begin{aligned}
& \text { Since } \lim _{n \rightarrow \infty} E\left\{\left|x_{n}-x\right|^{p}\right\}=0 \\
& , \lim _{n \rightarrow \infty} E\left\{\left|y_{n}-y\right|^{q}\right\}=0 \\
& \lim _{n \rightarrow \infty} E\left\{\left|x_{n}\right|^{p}\right\}=E\left\{|x|^{p}\right\}
\end{aligned}
$$$$
\text { and }
$$

by

$$
\text { using }
$$

theorem 
$E\left\{\left|x_{n} y_{n}-x y\right|\right\}=E\left\{\left|x_{n} y_{n}-x_{n} y+x_{n} y-x y\right|\right\}$

$\leq E\left\{\left|x_{n}\left(y_{n}-y\right)\right|\right\}+E\left\{\left|y\left(x_{n}-x\right)\right|\right\}$

$\left.\left.\leq \sqrt[p]{E\left\{\left|x_{n}\right|^{p}\right.}\right\} \sqrt[q]{E\left\{\left|y_{n}-y\right|^{q}\right.}\right\}$

$\left.+\sqrt[q]{E\left\{\left.y\right|^{q}\right.}\right\} \sqrt[p]{E\left\{x_{n}-\left.x\right|^{p}\right\}} \rightarrow 0$,

\section{$\underline{\text { Theorem (3-5) }}$}

Assume that $\left\{x_{n}\right\}$ be an uncertain variables sequence and $x$ be an uncertain variable. If

\section{Proof:}

By using theorem (1-6) then for any given numbers $\varepsilon>0$ we get $M\left[\left|x_{n}-x\right| \geq \varepsilon\right] \leq \frac{E\left[\left|x_{n}-x\right|^{p}\right]}{\varepsilon^{p}} \quad$ then $\lim _{n \rightarrow \infty} M\left[\left|x_{n}-x\right| \geq \varepsilon\right] \leq \lim _{n \rightarrow \infty}\left(\frac{E\left[\left|x_{n}-x\right|^{p}\right]}{\varepsilon^{p}}\right)$

\section{$\underline{\text { Example(3-9) }}$}

The convergence uncertain sequence in measure does not be convergence uncertain sequence in $p$ th moment. for example, Let $(\Omega, F, M)$ to be $\left\{y_{1}, y_{2}, \ldots\right\}$ such that $M\left(y_{k}\right)=\frac{1}{k}$ for $k=1,2,3, \ldots$. , and define the uncertain variables

by
As $n \rightarrow \infty, \quad$ then we get $\lim _{n \rightarrow \infty} E\left\{\left|x_{n} y_{n}-x y\right|\right\}=0$

$x_{n} \rightarrow x$ as $n \rightarrow \infty$ (in $p$ th moment ), then $x_{n} \rightarrow x$ as $n \rightarrow \infty$ (in measure) .

Which means that $\left\{x_{n}\right\}$ is converges to uncertain variable $x$ in measure.
$X_{n}\left(y_{k}\right)=\left\{\begin{array}{cc}n & , \text { if } n=k \\ 0 & , \text { o.w }\end{array}\right\}$ for $n=1,2,3, \ldots$. and $X=0$, then for any small number $\varepsilon>0$ we get that $\lim _{n \rightarrow \infty} M\left[\left|X_{n}-X\right| \geq \varepsilon\right]=\lim _{n \rightarrow \infty}\left(\frac{1}{n}\right)=0$, but $E\left[\left|X_{n}-X\right|\right]=1$

Since

$$
\begin{aligned}
& E\left[\left|X_{n}-X\right|^{p}\right]=\int_{0}^{\infty} M\left(\left|X_{n}-X\right|^{p} \geq r\right) d r \\
& \geq \int_{0}^{\infty} M\left(\left|X_{n}-X\right| \geq r\right) d r=E\left(\left|X_{n}-X\right|\right)=1
\end{aligned}
$$

Thus, $\lim _{n \rightarrow \infty} E\left[\left|X_{n}-X\right|^{p}\right]=1 \neq 0$. 


\section{4-Conclusion}

We obtained in this present paper some properties of expected value and several inequalities that based on expected value and uncertain measure, which is analogous

\section{Reference}

[1] Guo H and Iwamura K. 2011., A sufficient and necessary condition of uncertainty distribution, Journal of Interdisciplinary Mathematics, Vol.13, No.8, pp.2599-2604.

[2] Liu B. 2007., Uncertainty Theory, 2nd ed, Springer -Verlag, Berlin.

[3] Liu B. 2009, Theory and practice of uncertain programming, 3ed., UTLAB.

[4] Liu B. 2009 , Some research problems in uncertainty theory, Journal of Uncertain Systems, Vol.3, No.1, PP.3-10.

[5] Liu B. 2011, Uncertainty theory., 4th ed ., UTLAB.

[6] Liu Y and Ha M. 2010, Expected Value of Functions of Uncertain Variable, inequality. Then by using this inequality, we discussed some mathematical properties of convergence in $p$ th moment for uncertain sequence.

Journal of uncertainty Systems, Vol. 4, No.3, PP.181-186.

[7] Liu B. 2010, Uncertainty Theory: A Branch of Mathematics for odeling Human Uncertainty Springer- Verlag, Berlin.

[8] You C. 2009, On the convergence of uncertain sequences, Mathematical and Computer Modeling, No.49, PP.482-487.

[9] You C and Yan L. 2016, Relationships among convergence concepts of uncertain sequence, Computer modeling and new technologies Vol.20, No.3, pp12-16.

[10] Zhang Z. 2011., Some discussions on uncertain measure, Fuzzy optimization and decision making, DOI 10.1007/s 10700-0109091-0. 


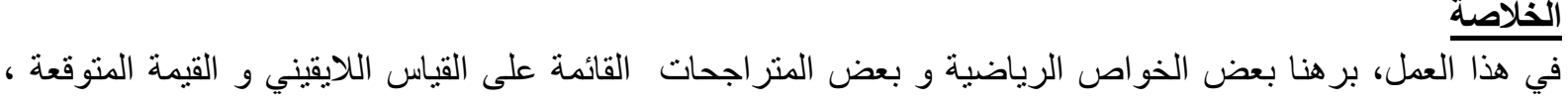
أخير ا حققنا العلاقة بين التقارب في (th moment p و التقارب في القياس للمتتابعات اللايقينية. الكلمات الالبلية الكيلية القياس اللايقيني ( غير المؤكد) ، المتغيرات اللايقينية، القيمة المنوقعة . 